CMAJ FACT SHEET

\title{
Fish oil and omega-3 fatty acids
}

\section{What are omega-3 fatty acids?}

Omega-3 fatty acids are polyunsaturated fatty acids found in certain foods. The term "omega- 3 " refers to their chemical structure. Omega-3 fatty acids cannot be made in the body and must be obtained from food; thus, they are essential fatty acids. There are many forms of omega-3 fatty acids, each with a different length and chemical structure.

\section{Where are omega- 3 fatty acids found?}

The shortest omega-3 fatty acid, $\alpha$-linolenic acid, is found in plants and plant oils, including leafy vegetables, walnuts, mustard seed oil, soy bean oil, canola oil and flaxseed oil (which is made up of $50 \% \alpha$-linolenic acid). The longer omega- 3 fatty acids, eicosapentaenoic acid (EPA) and docosahexaenoic acid (DHA), are found in algae, which are eaten by fish and baleen whales. We obtain these fatty acids by eating fish (e.g., salmon). Eicosapentaenoic acid and docosahexaenoic acid are often referred to as being from marine or fish sources.

\section{Are plant omega- 3 fatty acids the same as fish omega-3 fatty acids?}

Not quite. Although their chemical structures are similar, they have important differences, which affect their metabolism in the body.

\section{Can our bodies convert plant omega-3 fatty acids to fish omega- 3 fatty acids?}

Not well. Although our bodies can convert about $5 \%$ of $\alpha$-linolenic acid to eicosapentaenoic acid, the level of eicosapentaenoic acid in blood does not rise when we eat $\alpha$-linolenic acid.

\section{What do omega-3 fatty acids do?}

Both omega-3 fatty acids from fish and plants appear to reduce the risk of some types of heart disease. However, the results of all studies have not been consistent. Some suggest that fish oil may reduce mortality among patients with coronary heart disease, possibly by stabilizing the heart's rhythm and by reducing blood clotting. We don't know as much about the effects of plant omega-3 fatty acids, but they appear to have similar actions in reducing coronary heart disease events.

\section{What are the benefits of taking fish-oil supplements?}

It is usually stated that omega-3 fatty acids from fish may reduce cardiac deaths, inflammatory disease, including rheumatoid arthritis and ulcerative colitis, and promote brain development and mental function.

Evidence for a benefit for fish oils in preventing coronary artery restenosis after angioplasty and rhythm abnormalities is less clear.

\section{Are there risks from taking fish- oil supplements?}

In general, no. However, at least I study has suggested a negative effect among patients with implantable cardioverter defibrillators (to treat a heartrhythm problem) who were not taking antiarrhythmic medications. There also may be negative effects among men with angina in terms of coronary heart disease. Again, these adverse events were reported among men who were not taking medication to reduce their blood pressure and stabilize their heart rhythm.

\section{Are fish-oil supplements recom- mended for specific conditions?}

Some doctors recommend fish-oil supplements to prevent or treat cardiovascular disease. Although fish oil does not reduce LDL cholesterol, it may be recommended to lower serum triglycerides. In addition, infant formula is supplemented with docosa- hexaenoic acid in Europe to enhance development of the central nervous system because the rapidly growing brain requires large amounts of this fatty acid.

\section{Are fish oils essential for human health?}

Many people believe this to be true, but officials are still divided. Vegetarians, who do not have an obvious source of fish oil in their diet, seem to have a low incidence of coronary heart disease. For certain functions, $\alpha$-linolenic acid from vegetable sources may be able to substitute for longer fatty acids, either directly or after partial conversion to eicosapentaenoic acid.

\section{Are there other sources of fish omega-3 fatty acids?}

As mentioned, $\alpha$-linolenic acid may substitute for eicosapentaenoic acid. Docosahexaenoic acid used in infant formula comes from algae. With globally declining fish stocks and post-Kyoto awareness of the size of the human footprint, studies that involve plant-derived omega- 3 fatty acids (e.g., $\alpha$-linolenic acid or docosahexaenoic acid, or both) are needed to see if they also produce the desired benefits.

\section{David J.A. Jenkins MD PhD \\ Andrea R. Josse MSc}

From the Department of Medicine, Divisions of Endocrinology and Metabolism (Jenkins) and the Clinical Nutrition and Risk Factor Modification Centre (Josse), St. Michael's Hospital, Toronto, Ont.; and the Department of Nutritional Sciences (Jenkins, Josse), University of Toronto, Toronto, Ont.

Competing interests: see www.cmaj.ca/cgi/content |full/178/2/157

Acknowledgements: We thank Joseph Beyene PhD, Paul Dorian MD MSc, Michael L. Burr MD DSc (Med), Roxanne LaBelle BSc, Cyril W.C. Kendall $\mathrm{PhD}$ and Stephen C. Cunnane PhD for their substantive input into this fact sheet.

Une version française de cet article est disponible à l'adresse www.cmaj.ca/cgi /content/full/I78/2/I50/DCI 